UDC 621.77.01

Dobrov I. V.

Semichev A. V.

\title{
IMPROVEMENT OF THE OPTICAL METHOD FOR RESEARCHING THE CONTACT STRESSES DURING THE FORGING
}

Introduction. Among various optical and visioplastic methods for studying the processes of deformation of materials during forging [1] - [6], the optical method for determining the distribution of contact stresses [7] is of particular interest. The advantages of this method include its clarity, reliability of the research results, which qualitatively coincide with the load cell measurements of contact stresses $[8,9]$. The essence of this method is shown in Fig. $1 a^{1}$ and consists in the following. A workpiece made of plasticine or other similar material is forged by the transparent puncheon with cylindrical holes located along the axis of symmetry of the contact surface of the puncheon at equal distances from each other. When forging, the holes of the puncheon are filled with the plastic mass of the deformable workpiece and the height of filling of each hole of the puncheon is proportional to the value of the contact pressure during forging at the location of this hole in the puncheon.
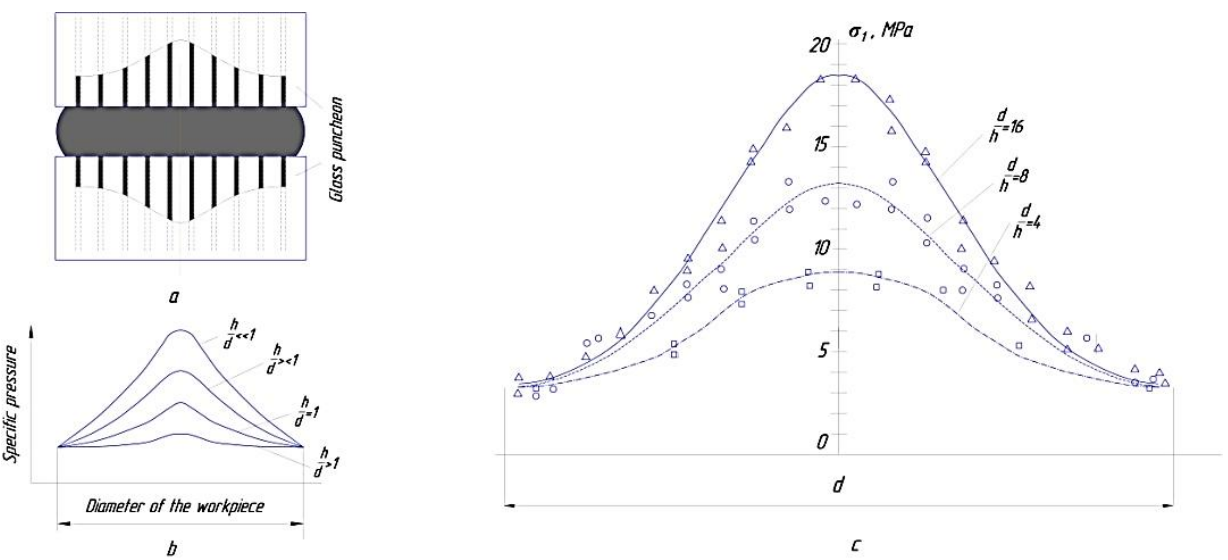

Fig. 1. Optical method for qualitative determination of the distribution of normal contact stresses during forging, proposed in the textbook:

$a$-flow of plastic mass (PMF) into the holes of glass puncheon; $b$ - diagrams of the heights of the PMF; $c$ - diagrams of normal stresses (method of load cells) during forging plastic metal samples [7]

The purpose of the work is to improve the optical method for studying contact normal stresses during forging of a symmetrical workpiece based on the analysis of data presented in the technical literature (distribution of normal contact stresses obtained by load cell method) and comparison the results of workpiece forging in a modernized puncheon with holes for flowing plastically deformable material.

Research. When analyzing the optical method of PMF the height of the flow of the plastic mass into the holes of the puncheon can be fixed only at a certain current time of the plastic deformation process. During static loading of a stationary puncheon, the optical method of PMF into holes in the puncheon is impracticable and it does not make sense. Consequently, distribution of contact normal stresses given in [6] (Fig. $1 c$ ), [8,9] during forging and their comparison with the PMF diagram in transparent puncheon (Fig. 1 a) must be carried out taking into account the influence of the dimensions of the workpiece in the diagrams of normal contact stresses (Fig. 2) in the process of deformation based on the following.

\footnotetext{
${ }^{1}$ In Fig. $1 a, b, c$ it is given data from work [7] edited by S. I. Gubkin [7]
} 


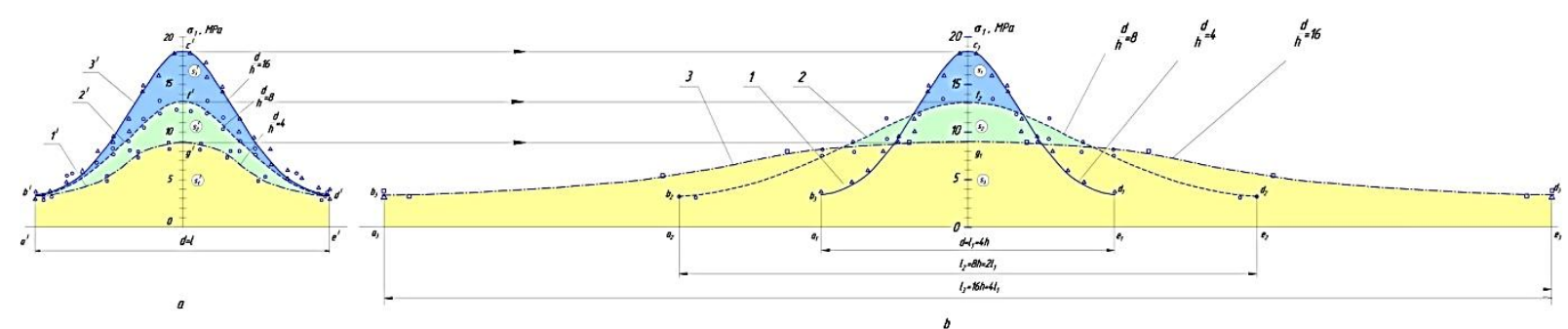

Fig. 2. Influence of the dimensions of the workpiece on the distribution of normal contact stresses under conditions of plane deformation of a symmetric workpiece:

$a$ - with the relative distribution of contact stresses without taking into account the deformation process; $b$ - with the relative distribution of contact stresses taking into account the current height of the deformed workpiece

Diagrams of contact normal stresses $1^{\prime}, 2^{\prime}, 3^{\prime}$ in Fig. 2 a correspond to similar diagrams $\frac{d}{h}=4, \frac{d}{h}=8$ and $\frac{d}{h}=16$ in Fig. $1 b$ ( $d$ - diameter (width $l$ during flat deformation) of workpiece, $h$ - height of workpiece). If $d=$ const then area $s_{1}^{\prime}$ of the diagram $1^{\prime}\left(a^{\prime} b^{\prime} c^{\prime} d^{\prime} e^{\prime}\right)$, colored blue at the moment $\left(t_{1}\right)$ during forging of workpiece to the height $h_{1}=\frac{d}{4}$ will be proportional to the force $P_{1}^{\prime} \approx s_{1}^{\prime}$. In its turn, the area $s_{2}^{\prime}$ of diagram $2^{\prime}\left(a^{\prime} b^{\prime} f^{\prime} d^{\prime} e^{\prime}\right)$, painted in light green color, at the moment $\left(t_{2}>t_{1}\right)$ of forging of workpiece to the height $h_{2}=\frac{d}{8}$ will be proportional to the force $P_{2}^{\prime} \approx s_{2}^{\prime}$, and area $s_{3}^{\prime}$ of the diagram $3^{\prime}\left(a^{\prime} b^{\prime} g^{\prime} d^{\prime} e^{\prime}\right)$, colored yellow at the moment $\left(t_{3}>t_{2}\right)$ of forging of workpiece to the height $h_{3}=\frac{d}{16}$ will be proportional to the force $P_{3}^{\prime} \approx s_{3}^{\prime}$. In this case from Fig $2 a$ follows that $P_{3}^{\prime} \approx s_{3}^{\prime}<P_{2}^{\prime} \approx s_{2}^{\prime}<P_{1}^{\prime} \approx s_{1}^{\prime}$. It is obtained that during plane deformation if height of specimens of equal diameters $\left(h_{3}>h_{2}>h_{1}\right)$ increases then force is decreases. This fact contradicts both the theory of working metals by pressure and the practice of specimen deformation during forging. To overcome this contradiction diagrams $1^{\prime}, 2^{\prime}, 3^{\prime}$ in Fig. $2 a$ are needed to be reduced to $1,2,3$ in Fig. $2 b$ based on the condition of plane deformation when the cross-sectional area of the original workpiece $S_{0}=h_{0} l_{0}$ does not change when forging the workpiece to the values: $h_{1}<h_{0}, h_{2}<h_{1}$ and $h_{3}<h_{2}$.

$$
S_{0}=h_{0} l_{0}=S_{1}=h_{1} l_{1}=S_{2}=h_{2} l_{2}=S_{3}=h_{3} l_{3},
$$

where $l_{0}, l_{1}, l_{2}$ and $l_{3}$ - respectively, the width of the workpiece at its heights $h_{0}, h_{1}, h_{2}$ and $h_{3}$. For diagrams $1^{\prime}, 2^{\prime}, 3^{\prime}$ in Fig. $2 a$ and diagrams $1,2,3$ in Fig. $2 b$ if the values $h_{1}, h_{2}$ and $h_{3}$ are equal and from (1) follows that in each moment of time of forging the height of the workpiece sequentially determines the current workpiece width: $l_{1}=d, l_{2}=2 l_{1}, l_{3}=2 l_{3}$. Accordingly, the diagrams $1\left(a_{1} b_{1} c_{1} d_{1} e_{1}\right), 2\left(a_{2} b_{2} f_{2} d_{2} e_{2}\right)$ and $3\left(a_{3} b_{3} g_{3} d_{3} e_{3}\right)$ define: area $s_{1}$, painted blue, area $s_{2}>s_{1}$, painted in light green, and the area $s_{3}>s_{2}$, painted yellow. Therefore, the diagrams 1 , 2,3 in Fig. $2 b$ reflect the actual conditions of the workpiece forging when the condition is met and these diagrams reflect the actual change in the distribution of contact normal stresses obtained by the load cell method during plane deformation of a symmetrical workpiece.

$$
P_{3} \approx s_{3}>P_{2} \approx s_{2}>P_{1} \approx s_{1} \text { if } h_{3}<h_{2}<h_{1}<h_{0} .
$$


Significant disadvantage of the optical PMF method (Fig. $1 a$ ) is that the height of the PMF in the puncheon holes depends on the friction of the workpiece material against the inner surfaces of the holes in the puncheon, which reduces the reliability of determining the PMF height to characterize the distribution of the magnitude of contact normal stresses during forging. This disadvantage is eliminated by using a composite puncheon, a schematic diagram of which is shown in Fig.2 $a$. A puncheon with a thickness $b$ consists of two symmetrical plates 2 and 3 with thickness $0,5 b$, connected by clamping screws 3 . In the plane of the puncheon connectors
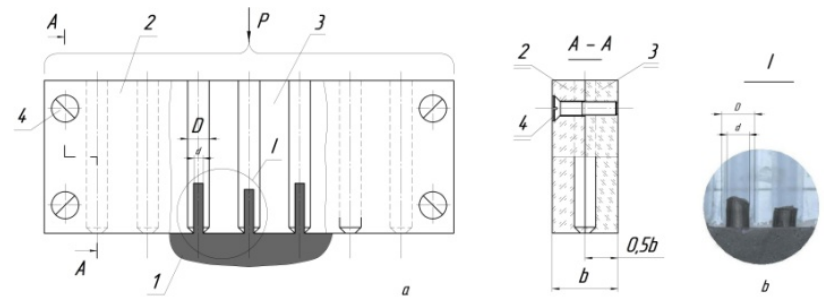

Fig. 3. Scheme of the modernized puncheon:

$a$ - assembled puncheon; $b$ - position of the workpiece in half of the disassembled puncheon

it is performed the holes with the diameters $d$ located symmetrically on the side of the contact surface of the workpiece and the puncheon, and with the diameters $D>d$ on the side of the surface of the puncheon to which the forging force is applied, providing the PMF of the workpiece 1 into the holes of the puncheon (Fig. $3 \mathrm{~b}$ ).

This design of the composite puncheon allows after the forging of the original workpiece, indicated in Fig. 4 by dash-dotted lines with dimensions: $h_{0,1} \times l_{0,1} \times b$ (Fig. $4 a$ ), $h_{0,2} \times l_{0,2} \times b$ (Fig. $4 b$ ), $h_{0,3} \times l_{0,3} \times b$ (Fig. $4 c$ ) to the final dimensions of forged workpieces
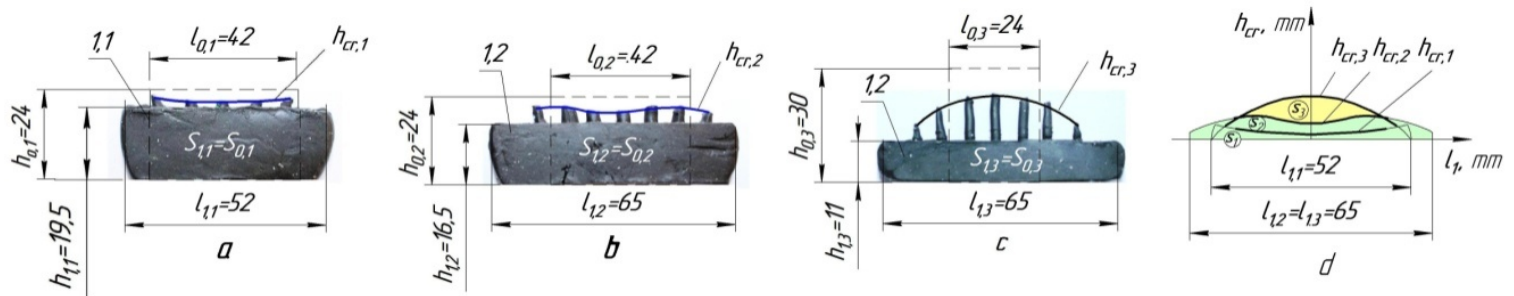

Fig. 4. Results of the research of the distribution of normal contact stresses during forging of a symmetrical workpiece made of plasticine under conditions of plane deformation: $a, b, c-$ pictures of forged workpieces from the original workpieces (dotted lines); $d$-comparison of the diagrams of the flow of plasticine into the holes of the composite puncheon

$h_{1,1} \times l_{1,1} \times b$ (Fig. $4 a$ ), $h_{1,2} \times l_{1,2} \times b$ (Fig. $4 b$ ), $h_{1,3} \times l_{1,3} \times b$ (Fig. $4 c$ ) remove the workpieces from the puncheon (Fig. $3 \mathrm{~b}$ ) when separating halves 2,3 of the composite puncheon without destroying the plastic material in the holes of the puncheon.

Experimental research of the distribution of normal contact stresses during the forging of a symmetric plasticine workpiece during plane deformation on a screw press using a prefabricated matrix containing a base and flat side surfaces, which are connected with each other using bolts at a distance $b$, which is equal to the width of the modernized puncheon. Fig. $4 a-c$ shows forged workpieces $1,1,1,2,1,3$ with areas: $S_{1,1}=h_{1,1} \times l_{1,1}=h_{0,1} \times l_{0,1}, \quad S_{1,2}=h_{1,2} \times l_{1,2}=h_{0,2} \times l_{0,2}$, $S_{1,3}=h_{1,3} \times l_{1,3}=h_{0,3} \times l_{0,3}$, which characterizes the observance of the condition of flat deformation of the workpiece in the process of forging of the workpieces and, accordingly, makes it possible to determine the height of the PMF of the workpiece 1 in puncheon holes, in the form of diagrams $h_{c r, 1}, h_{c r, 2}, h_{c r, 3}$, reflecting the nature of the distribution of contact normal stresses, depending on the deformation value of the workpiece. 
Analysis of research results. In Fig. $4 d$ comparative diagrams $h_{c r, 1}, h_{c r, 2}, h_{c r, 3}$ of PMF of the workpiece into the holes of the puncheon at plane deformation of the symmetrical workpiece are given. Area ratios $s_{1}<s_{2}, s_{2}<s_{1}$ of the diagrams $h_{c r, 1}, h_{c r, 2}, h_{c r, 3}$ confirms (2) the correspondence between the nature of the distribution of the PMF diagrams of the workpiece in the holes of puncheon and the diagrams of the distribution of contact normal stresses obtained by the method of load cell research of workpieces from various materials. It is shown that in the process of forging there is a constant redistribution of contact normal stresses at the beginning of plastic deformation. The normal stress diagram tends to have a slight crowning on axis of the symmetry of the workpiece. With an increase in the degree of deformation, the stress diagram represents a curve, which has two symmetric crowns shifted from the workpiece symmetry axis to the sides of the workpiece side surfaces free from the load, and with significant deformation, the normal contact stress diagram forms a shape symmetric about the workpiece symmetry axis.

\section{CONCLUSIONS}

When comparing with each other the combined graphs of the distribution of contact normal stresses along the axis of symmetry of the workpiece, presented in the technical literature, depending on the ratio of the position of the considered section of the workpiece relative to the length (diameter) of the contact surface, it is necessary to take into account the real change in the dimensions of the contact surface during forging of the original workpiece with the different degrees of deformation.

An optical method for studying the distribution of normal contact stresses has been improved, based on the flow of plastic material of the workpiece into the holes of the puncheon during forging of a symmetric workpiece under plane deformation, which makes it possible to expand the physical understanding of the mechanism of redistribution of contact normal stresses in the process.

\section{REFERENCES}

1. Markov O.E., Aliiev I.S. Resource-saving technological processes for forging large forgings from ingots: monograph. Kramatorsk: DSEA. 2012. 324 p. (in Russian).

2. Kuzmina O.M. Application of the method of rods to the analysis of the stress-strain state during upsetting. Improving the processes and equipment of working by pressure in mechanical engineering and metallurgy. Kramatorsk: DSEA. 2004, pp. 381-383. (in Russian).

3. Dobrov I.V., Semichev A.V., Getman I.I. A review of optical methods for determining the kinematic parameters of plastic deformation of a symmetric workpiece during upsetting. Theoretical and practical problems in the processing of materials by pressure and the quality of special education: Proceedings of the International scientific-technical. conf., 14-18 December 2015. Kyiv. 2015, pp. 59-61. (in Russian).

4. Thomsen E., Young Ch., Kobayashi Sh. Mechanics of plastic deformations in metal processing. Moscow: Mechanical Engineering. 1968. 504 p. (in Russian).

5. Dobrov I.V., Semichev A.V., Novikov B.O., Getman E.V. Development of a new visioplastic method for studying the metalforming processes using the example of upsetting a symmetrical workpiece. Materials Working by Pressure. Kramatorsk: DSEA. 2017. 1 (44), pp. 44-48. (in Russian).

6. Pat. 10887 Ukraine. G01B11/16. Method for determining the kinematics of the plastic deformation process. Dobrov I.V., Semichev A.V., Getman I.I. 2016.

7. Gubkin S.I. Plastic deformation of metals. Moscow: Metallurgizdat. 1961. 2, 416 p. 1955. $280 \mathrm{p}$

8. Unksov E.P. Engineering methods for calculating forces in the processing of metals by pressure. Mashgiz.

9. Grudev A.P., Zilberg Yu.V., Tilik V.T. Friction and lubrication in metal forming. Moscow: Metallurgy. 1982. 312 p.

Dobrov I. V. - D-r Sc. (tech), Prof. of NMetAU;

Semichev A. V. - Cand. of tech. Sc., Assist. Prof. of NMetAU.

NMetAU - National Metallurgical Academy of Ukraine, Dnipro.

E-mail: igordobrov@yahoo.com 